\title{
Some Remarks about CD147
}

\section{Bubnovskaya Larissa $\mathbf{N}^{*}$}

Department of Metastatic Problems of Tumor Microenvironment, R.E. Kavetsky Institute of Experimental Pathology, Oncology and Radiobiology of National Academy of Science of Ukraine, Ukraine

*Corresponding Author: Bubnovskaya Larissa N, Department of Metastatic Problems of Tumor Microenvironment, R.E. Kavetsky Institute of Experimental Pathology, Oncology and Radiobiology of National Academy of Science of Ukraine, Ukraine.

DOI: 10.31080/ASCB.2020.04.0241

In December 2019, the novel coronavirus (CoV), SARS-CoV-2, has emerged in China. The disease caused by SARS-CoV-2 was named as COVID-19 by the World Health Organization (WHO). The emergence of SARS-CoV-2 and its rapid spread across the world has triggered a global health emergency. Nevertheless, so far there exist no specific antiviral drugs for treatment of the disease, which poses great challenge to control and contain the virus The pathological features of patients with COVID-19 are very similar to those of patients with SARS and MERS (Middle East Respiratory Syndrome) infection. However, COVID-19 is more contagious than SARS and MERS.

So, what does COVID-19 mean for patients with cancer, their physicians, and the wider oncology discipline? The pandemic has altered the routine for oncology patients. Oncology societies and national authorities have been quick to issue guidelines on cancer care during the pandemic. In its guidance for managing patients with cancer during this epidemic, in addition to better protection, need online medical counseling, appropriate identification and acute treatment of critical cases. Patients with cancer are facing unprecedented circumstances. "On the one hand, a patient might be at high risk of contracting the infection and dying from it; on the other hand, the patient might be at high risk of the cancer progressing or causing death if it is not treated appropriately" said Richard Schilsky, chief medical officer of the American Society of Clinical Oncology (ASCO).

Patients with cancer are a high-risk group in the COVID-19 and with a poorer prognosis than those without cancer. Accumulated evidence has shown that development of cancer is usually associated with a blunted immune status characterised by overexpressed immunosuppressive cytokines, suppressed induction of proinflammatory danger signals, impaired dendritic cell maturation, and enhanced functional immunosuppressive leukocyte populations, which is contradictory to the events believed to result in severe events in patients with COVID-19. They are already vulnerable to infection because of their underlying illness, and very often immunosuppressed status, and are at increased risk of developing severe complications from the virus, including intensive care unit admission or even death. However, overall, current evidence remains insufficient to explain a conclusive association between cancer and COVID-19.

The outbreak of coronavirus disease 2019 (COVID-19) is of international concern. To the best of our knowledge, the Comment from Wenhua Liang and colleagues published in The Lancet Oncology on Feb 14, 2020, was the first to focus on COVID-19 infection in patients with cancer.

It was proposed three major strategies for patients with cancer in this COVID-19 crisis, and in future attacks of severe infectious diseases. First, an intentional postponing of adjuvant chemotherapy or elective surgery for stable cancer should be considered in endemic areas. Second, stronger personal protection provisions should be made for patients with cancer or cancer survivors. Third, more intensive surveillance or treatment should be considered when patients with cancer are infected with SARSCoV-2, especially in older patients or those with a number of comorbidities, very often with such as hypertension, heart disease, chronic kidney disease, diabetes, etc. who are more likely to develop severe disease.

While our knowledge of COVID-19 was still developing and attention should be given how SARS-CoV-2 penetrates into the cell. It was decided that SARS-CoV-2, like its precursor the etiological agent of severe acute respiratory syndrome (SARS), an acute pulmonary syndrome, SARS-CoV binds with functional receptor for SARS-CoV angiotensin-converting enzyme 2 (ACE2) on the host cell membrane with high affinity and which regulates both the crossspecies and human-to-human transmissions of SARS-CoV. Several lines of evidence suggest that ACE2 is a physiologically relevant and widely recognized essential receptor in virus invasion, and viral replication during infection. However, four surface peptides of SARS-CoV-2 give the chance to assume some more targets as points of binding. It was reported by Chinese scientists a research finding that SARS-CoV-2 can also enter host cells via novel route of CD147-spike protein (SP). SP bound to CD147 (also called Basigin), a receptor on the host cells, thereby mediating the viral invasion elucidate the invasive mechanisms of SARS-CoV-2, thereby 
providing a potential target for developing specific antiviral drugs. CD147 is needed special attention in oncology because it has been documented to be highly expressed in various cancer cells and mostly localized in the cell membrane with very limited expression in normal tissues. CD147 is associated with assembly of numerous pro-oncogenic proteins in the plasma membrane and may play a fundamental role in properties characteristic of cancer stem-like cells. CD147 has already been described to facilitate HIV, measles virus, and malaria entry into host cells. CD147 is a widely distributed transmembrane glycoprotein, belonging to the immunoglobulin superfamily, plays fundamental roles in intercellular interactions in numerous pathological and physiological processes and can function as a key mediator of inflammatory and immune responses.

Increased expression of CD147 is associated with pathogenesis of a number of diseases, such as asthma-mediated lung inflammation, rheumatoid arthritis, multiple sclerosis, myocardial infarction and ischemic stroke, Alzheimer's disease and cancer. Accumulating evidence has demonstrated also that CD147 expression correlate with worse prognosis across many cancer types, including malignant melanoma, liver cancer, lung cancer, breast cancer, head and neck cancer, cervical cancer, hepatocellular carcinoma, colon cancer and orchestrates tumor cell proliferation, stimulates fibroblasts to produce a large number of matrix metalloproteinases (MMPs) leading to tumor cell invasion, plays pivotal roles in intercellular interactions involved in tumor metastasis and angiogenesis and tumor cell glycolysis through critical molecules such as monocarboxylate transporters, MMPs, especially under hypoxic conditions, and multidrug resistance. It has been implicated in a variety of physiological and pathological activities through interacting with multiple partners, including cyclophilins, monocarboxylate transporters, caveolin-1, and integrins. Aforementioned testify that over-expression of CD147 is associated with various tumors, and very often with pathogenesis of a number of diseases that are a common comorbidity in patients with malignancy and it seems to promote SARS-CoV-2 enter into the host cells. That is why cluster of differentiation 147 (CD147) gains so high value for patients with cancer and may serves as unifying target for reducing its overexpression. For the moment, there are appeared the remarkable achievements of investigators rapidly generating scientific data. Recent novel studies have demonstrated the potential application of CD147 not only as a potential diagnostic marker but also as an effective therapeutic target for cancer chemotherapy. Exciting clinical progress has been made in treatment using CD147-directed monoclonal antibodies. CD147 is recognized as an effective therapeutic target for hepatocellular carcinoma and other cancers. The therapeutic potential of monoclonal antibodies has been well recognized in the treatment of many diseases. Moreover, CD147 is recognized also as a potential target for developing specific antiviral drugs: monoclonal antibodies are capable of providing selective therapy by selecting for extracellular or transmembrane proteins that are highly expressed on tumor cells with limited expression on normal cells. It was shown that anti-CD147 antibody could competitively inhibit CD147 expression and prevent the viruses from invading host cells. Therefore, additional independent large-cohort studies are needed. Hopefully these approaches will be approved into clinical trials to benefit the oncological patients. Until the COVID-19 pandemic is over, remains a high priority the development specific therapeutic agents by the oncology community.

\section{Assets from publication with us}

- Prompt Acknowledgement after receiving the article

- Thorough Double blinded peer review

- Rapid Publication

- Issue of Publication Certificate

- High visibility of your Published work

Website: https://www.actascientific.com/

Submit Article: https://www.actascientific.com/submission.php Email us: editor@actascientific.com

Contact us: +919182824667 